\title{
Detection of chemical alterations at internal walls of microchannel flow cells by nondestructive fluorescence depolarization
}

\author{
Cristina M. Quintella, ${ }^{\mathrm{a}, *}$ Ângelo M.V. Lima, ${ }^{\mathrm{a}}$ Alaíde P. Mammana, ${ }^{\mathrm{b}}$ Marcos A. Schreiner, \\ Iuri Pepe, ${ }^{\mathrm{c}}$ and Yuji N. Watanabe ${ }^{\mathrm{a}}$ \\ a Instituto de Química, Universidade Federal da Bahia, Campus de Ondina, 40170-290 Salvador, BA, Brazil \\ ${ }^{\mathrm{b}}$ Centro de Pesquisas Renato Archer-CenPRA, Rod. D. Pedro I, km 143.6, Amarais, 13082-120 Campinas, Brazil \\ c Instituto de Física, Universidade Federal da Bahia, 40170-290 Salvador, BA, Brazil
}

Received 18 March 2003; accepted 19 September 2003

\begin{abstract}
A recent trend is the production of workable microchannel flow cells (MF cells). The nondestructive methods used to assess their reliability are based mainly on output monitoring and do not evaluate internal chemical interactions. We investigate a nondestructive method for evaluating changes in the chemical composition of the inner walls based on evaluation of the extent of alignment of a fluorescent probe in a liquid flowing within MF cells. Two MF cells were built with a $10-\mu \mathrm{m}$ inner spacing. Their inner walls had four parallel $\mathrm{SnO}_{2}$ strips, $2.00 \mathrm{~mm}$ wide, separated by $0.50-\mathrm{mm}$-wide glass strips. One cell had strips parallel to the flow and the other perpendicular. Flow-induced intermolecular alignment of rhodamine B in monoethylene glycol was scanned with $28-\mu \mathrm{m}$ precision by fluorescence depolarization, using polarized-laser-induced fluorescence within induced flows (PLF-FI). No changes of polarization were seen when the flow was stopped. Under flowing conditions, polarization was always $4 \%$ lower in the glass region as compared to $\mathrm{SnO}_{2}$. Glass had a higher solid-liquid interfacial tension (determined by contact angle measurements), thus being more wettable and increasing the drag, which propagates into the liquid flow, decreasing polarization. PLF-FI can thus identify regions with different chemical constitutions.
\end{abstract}

(C) 2003 Elsevier Inc. All rights reserved.

Keywords: Fluid microdynamics; PLF-FI; Interfacial tension; Wetting; Adhesion; Drag; Dynamic interactions; Liquid flow

\section{Introduction}

Fluid mechanics usually deals with flows in systems much thicker than the boundary layer width. In several analytical, chemical, and biological systems, flow occurs inside microchannels whose width is comparable to that of the boundary layer itself [1], where concepts such as fully developed laminar and turbulent regime do not apply. Thus, molecular hydrodynamics becomes important for processes within microchannel flow cells (MF cells) due to the strong interaction between the microchannel walls and the molecules of the flowing liquid. Microfluidics is present within chromatographic columns, as well as nanodevices and geological reservoirs, and is currently of great technological interest in capillary electrophoresis, where microchannel width can be below $50 \mu \mathrm{m}$.

\footnotetext{
* Corresponding author.

E-mail address: cristina@ufba.br (C.M. Quintella).
}

Interfacial tension $\left(\Gamma_{\mathrm{SL}}\right)$ is commonly used to evaluate and establish control parameters for wall-liquid interactions important in molecular hydrodynamic processes. Static $\Gamma_{\mathrm{SL}}$ have been determined for several decades by sessile drop contact angle $\left(\theta_{c}\right)$ measurements [2]. There is a causal relation between the advancing $\theta_{c}$ and receding $\theta_{c}$ and the interfacial chemical groups present at the interface, as well as their relative orientation $[3,4]$.

Recently, fluorescence depolarization was applied for the first time to evaluate dynamic $\Gamma_{\mathrm{SL}}$ of liquids flowing as thin sheets on two flat surfaces with different chemical constitutions [5].

Fluorescence spectroscopy usually monitors samples as a function of excitation and/or emission wavelength at a single spot and without polarization resolution. Fluorescence depolarization mapping requires neither temporal nor wavelength resolution. What is crucial is to determine polarization of the fluorescence. Laser excitation is chosen in order to improve fluorescence detection and polarized fluorescence is detected 
without need of wavelength resolution. The technique used is polarized laser induced fluorescence (PLF).

The principles of PLF have been discussed before [6]. Briefly, vertically polarized laser light excites fluorescent probes within a sample. Their absorption is proportional to the cosine square of the angle between the molecular transition dipole moment and the laser electric field. If the transition moment is parallel to the longitudinal molecular axis (as for rhodamine, the probe used in this work), primarily the nearly vertical molecules will absorb laser radiation. During their excited state lifetime, photoselected probes rotate as a function of their mobility, which depends on chemical environment. In PLF fluorescence emission is discriminated according to its polarization and compared with the incident laser polarization.

For a fluorescent molecule to be an intermolecular alignment probe, its rate of rotation (rotational diffusion time) must be approximately equal to its excitation lifetime. Otherwise, the probe either would be nearly rotationally immobile during its excited lifetime or would be completely depolarized by rotational diffusion.

Fluorescence depolarization data can be interpreted either as a bidimensional phenomenon in terms of polarization $(\mathrm{P})$, or as a tri-dimensional phenomenon in terms of anisotropy (r) $[7,8]$ :

$P=\frac{I_{\mathrm{II}}-I_{\perp}}{I_{\mathrm{II}}+I_{\perp}}, \quad r=\frac{I_{\mathrm{II}}-I_{\perp}}{I_{\mathrm{II}}+2 I_{\perp}}, \quad r=\frac{2 P}{3-P}$.

For fluorescence depolarization within liquid flows (PLF-FI), the high velocity imposed by the liquid flow causes a preferential molecular orientation in the downstream direction that is photoselected by the laser. Polarization and anisotropy are maximal if molecules are still in the downstream direction when they fluoresce.

For a thin liquid layer flowing on a solid surface, polarization and anisotropy will be high when the solid-liquid dynamic $\Gamma_{\mathrm{SL}}$ is low. Since the drag at the interface is small, increasing the molecular slip, the molecular domains [9] will remain aligned with the flow. If dynamic $\Gamma_{\mathrm{SL}}$ increases, the vicinal flowing layer establishes stronger transient intermolecular forces, increasing the drag and decreasing the slip, and turbulence will propagate throughout the liquid, misaligning flowing molecular domains and causing both polarization and anisotropy to decrease.

PLF-FI has already been applied to liquid flows outside microchannels. Free thin liquid jets in air were mapped with respect to their nonhomogeneous intermolecular orientation in the central region $[10,11]$. Fluorescence depolarization kinetics was obtained with picosecond resolution within certain regions, indicating a strong anisotropy in $\theta$ and $\phi$ rotational diffusion dynamics [12]. Previous results have shown that for thin channels the flow consists mainly of superposed boundary layers where interfacial chemical interactions become predominant over bulk flow [13]. Recently, PLF-FI was used to evaluate relative dynamic $\Gamma_{\mathrm{SL}}$ for monoethylene glycol flowing on either glass or tin dioxide and it was found that dynamic $\Gamma_{\mathrm{SL}}$ was stronger for glass than for tin dioxide [5].

The aim of this paper is to evaluate PLF-FI as a reliable nondestructive method for observing alterations of chemical constitution at inner walls of MF cells. For this purpose, we carried out bidimensional measurements of fluorescence depolarization in MF cells (10 $\mu \mathrm{m}$ thick) with inner walls made of stripes with two different constitutions. The results of change in flow orientation (parallel and perpendicular to the stripes) were also reported and analyzed.

As far as we are aware, this is the first time that PLF-FI has been used as a nondestructive method for visualizing changes in the chemical constitution of the inner walls of MF cells, as well as their sensitivity to orientation with respect to the flow.

\section{Materials and methods}

Four $40 \times 40 \mathrm{~mm}$ soda-lime glass slides were coated with 2.00 -mm-wide stripes of tin dioxide $\left(\mathrm{SnO}_{2}\right)$ that were $0.010 \mu \mathrm{m}$ thick and separated from one another by $0.50 \mathrm{~mm}$. Their production was similar to that of liquid crystal displays made on the CenPRA preindustrial assembly line [14]. Atomic force microscopy (AFM) showed that both glass and $\mathrm{SnO}_{2}$ were quite flat, with roughness below $10 \mathrm{~nm}$.

Two microchannel flow cells (MF cells) were constructed by juxtaposing two of the slides (Fig. 1). They were spaced by spreading cylindrical spacers $(10 \mu \mathrm{m}$ in diameter) over one of the slides prior to closing the cell (about 40 spacers per $\mathrm{cm}^{2}$ ). In both cells, the $\mathrm{SnO}_{2}$ stripes on the inner face of one slide matched those on the other face. In one cell (MF cell A) to the stripes were oriented perpendicular to the liquid flow and in the other (MF cell B) parallel to it.

The flow was produced in aspirated mode by a fourchannel Gilson MP4 peristaltic pump (Pp) at $30 \mathrm{rpm}$. The flowing liquid was monoethylene glycol (MEG) from Synth (batch 50070, 99.5\% purity). A volume of $15 \mathrm{ml}$ was pumped at $(24.5 \pm 0.5)^{\circ} \mathrm{C}$ in a closed loop of 3.6-mminner-diameter polypropylene tube, producing a flow of $39 \mathrm{~mL} \mathrm{~s}^{-1}$, corresponding to a velocity of $9.75 \times 10^{-1} \mathrm{~m} \mathrm{~s}^{-1}$ within the MF cell.
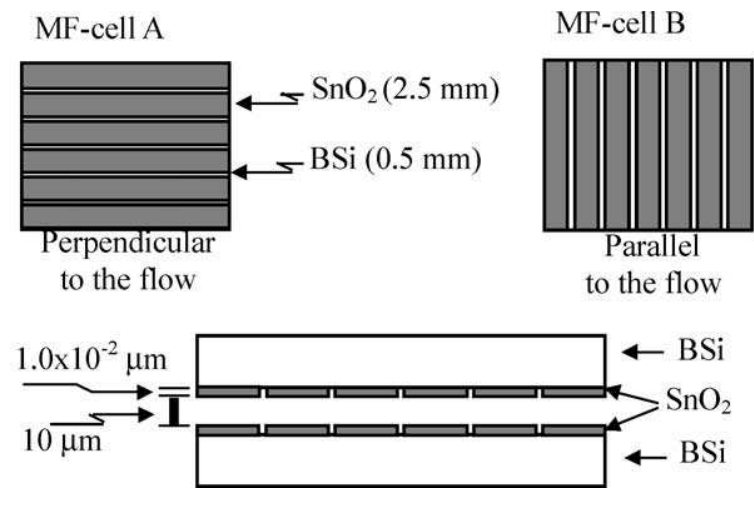

Fig. 1. Scheme of the two microchannel flow cells (MF cells). 
Rhodamine B (RB) from Merck (99.99\% purity) was used as the fluorescent probe. Its concentration was chosen to avoid fluorescent aggregates that might serve as additional acceptors of the excitation energy or favor nonradiative excitation transport. Rhodamine B dissolved in ethylene glycol forms dimers at concentrations above $10^{-2} \mathrm{molL}^{-1}$ [15]. This value is two orders of magnitude higher than in water or methanol, which is attributed to the stronger repulsion between positively charged moieties in ethylene glycol. Förster nonradiative energy transfer has a critical radius smaller than the average distance between rhodamine molecules in our system.

PLF requires that the probe rotational diffusion time be of the same order of magnitude as its excited state lifetime. It is reported in the literature that an increase in solvent viscosity increases both the probe fluorescence lifetime $[16,17]$ and the rotational relaxation time [18], which is the case for MEG relative to water. Concentrations between $1.8 \times 10^{-3}$ and $2.2 \times 10^{-3} \mathrm{~mol} \mathrm{~L}^{-1}$ were chosen in order to satisfy all these constraints on the fluorescent probe and obtain reliable signal intensity.

Fluorescence from dye adsorbed to the walls or present in the solvent layers vicinal to the wall may differ in its properties from that of dye molecules in the bulk. However, the contribution due to fluorescence from rhodamine interacting with the microchannel inner walls did not exceed $5 \times 10^{-4}$ $(0.05 \%)$. This estimate is based on an assumed thickness of the vicinal solvent layer of $2 \times 25 \AA$ as compared to an optical path length of $10 \mu \mathrm{m}$. Absorption spectra obtained prior and after the experiment showed that no rhodamine was left on or absorbed by either glass or $\mathrm{SnO}_{2}$ surfaces.

Fig. 2 shows the optical path for polarized laser-induced fluorescence (PLF) used to evaluate fluorescence depolarization. Briefly, a 532-nm Nd:YAG laser beam (Continuum Surelite SLII-10) at $0.55 \mathrm{~mJ}$ is deflected by mirror M and focused by lens L1 into the sample, on a $2.4-\mu \mathrm{m}$ diameter. A vertical polarizer P1 ensures $100 \%$ polarization. Fluorescence is collected by lens L2 at $40^{\circ}$ to the incident laser beam and passes through a 550 -nm cut-off filter $\mathrm{F}$ to block laser radiation. Vertical and horizontal fluorescence polarizations were selected by manually rotating polarizer P2. Polarized fluorescence reaches detector PD at a solid angle of 0.02 sr. This detector, of in-house design, consists of a BPW-2/RS-Electronics photodiode with a $7.5-\mathrm{mm}^{2}$ active area and operates as a current-to-voltage converter with two optional sensitivity ranges. The PD output was connected to a data acquisition and control interface (I) coupled to a personal computer (PC). The laser pulse has a half-width of $7 \mathrm{~ns}$ and the rhodamine fluorescence lifetime is $\sim 5 \mathrm{~ns}$ under our experimental conditions [19]. An active area RC low filter (Int) with a time constant covering about 10 laser pulses was employed to integrate the fluorescence signal. The interface was developed to dialogue with the parallel printer port. It consisted of two 12-bit ADCs (ADS 7804 from Burr Brown), two stepping motor control units, and one 4-channel micro-valve control unit.

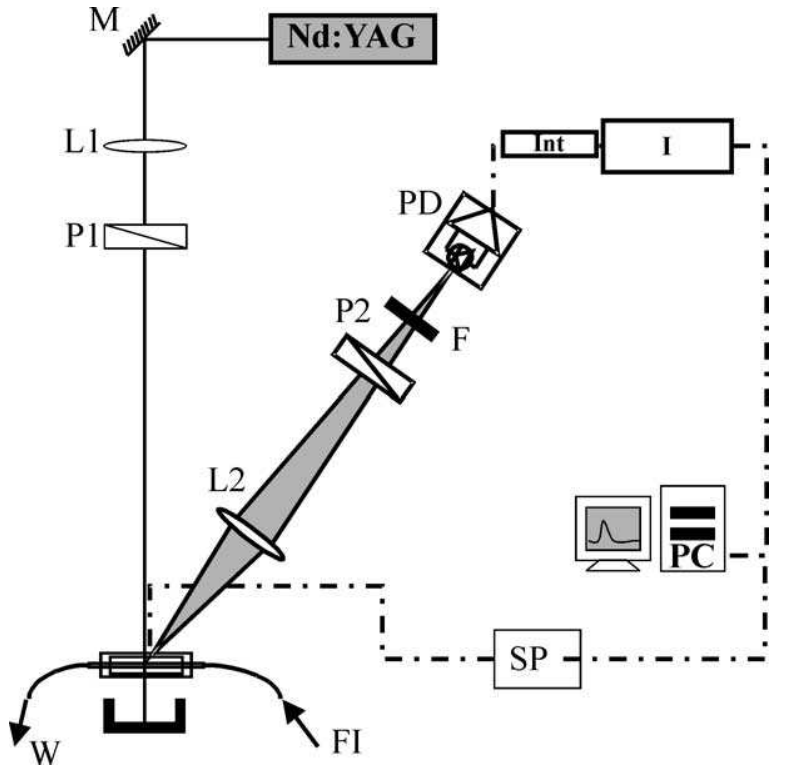

Fig. 2. Experimental setup for polarized laser-induced fluorescence within liquid-induced flows (PLF-FI) to detect fluorescence depolarization. Nd:YAG-laser, M-mirror, L1, L2-lenses, P1, P2-Glan-Thompson polarizer, $\mathrm{PD}$ - photodiode, Int - integrator, I-interface, $\mathrm{PC}$ - personal computer, $\mathrm{SP}$ - sample positioning system, $\mathrm{FI}$ - liquid flow, $\mathrm{W}$ - waste.

The MF cell position was varied uniformly in relation to the laser beam direction by a two-axis translation frame [20] based on two stepping motors and remotely controlled by software written in Q-BASIC that permits sample positioning and real time data acquisition. The device had mechanical repeatability of better than $0.1 \%$.

\section{Results and discussion}

MF cells were frontally scanned by acquiring profiles along the width with $28-\mu \mathrm{m}$ resolution at four height positions. Each profile (vertical and horizontal) was mapped at least five times and then averaged prior to calculation of polarization and anisotropy in order to avoid random noise. Since the MF cell spacers are randomly spread, their contributions are similar for both inner walls regions.

No signal was detected for scans with flowing MEG without $\mathrm{RB}$, ruling out the possibility that the signal was due to laser scattering at the MF cells interfaces. When the flow was stopped, the polarization was $(3 \pm 1) \%$ and the profiles did not present any differences between the glass and the $\mathrm{SnO}_{2}$ regions of the inner walls within our experimental precision.

The static contact angles $\left(\theta_{c}\right)$ of sessile MEG drops were $(32 \pm 2)^{\circ}$ and $(44 \pm 2)^{\circ}$ on glass and $\mathrm{SnO}_{2}$, respectively, corresponding to $\cos \theta_{c}$ of $(0.85 \pm 0.01)$ and $(0.72 \pm 0.01)$. The larger $\theta_{c}$ on $\mathrm{SnO}_{2}$ than on glass confirms the higher $\Gamma_{\mathrm{SL}}$ of the glass regions.

Fig. 3 shows profiles (bottom) and polarization maps (top) obtained for cell A (stripes perpendicular to flow) and cell B (stripes parallel to flow), respectively. 

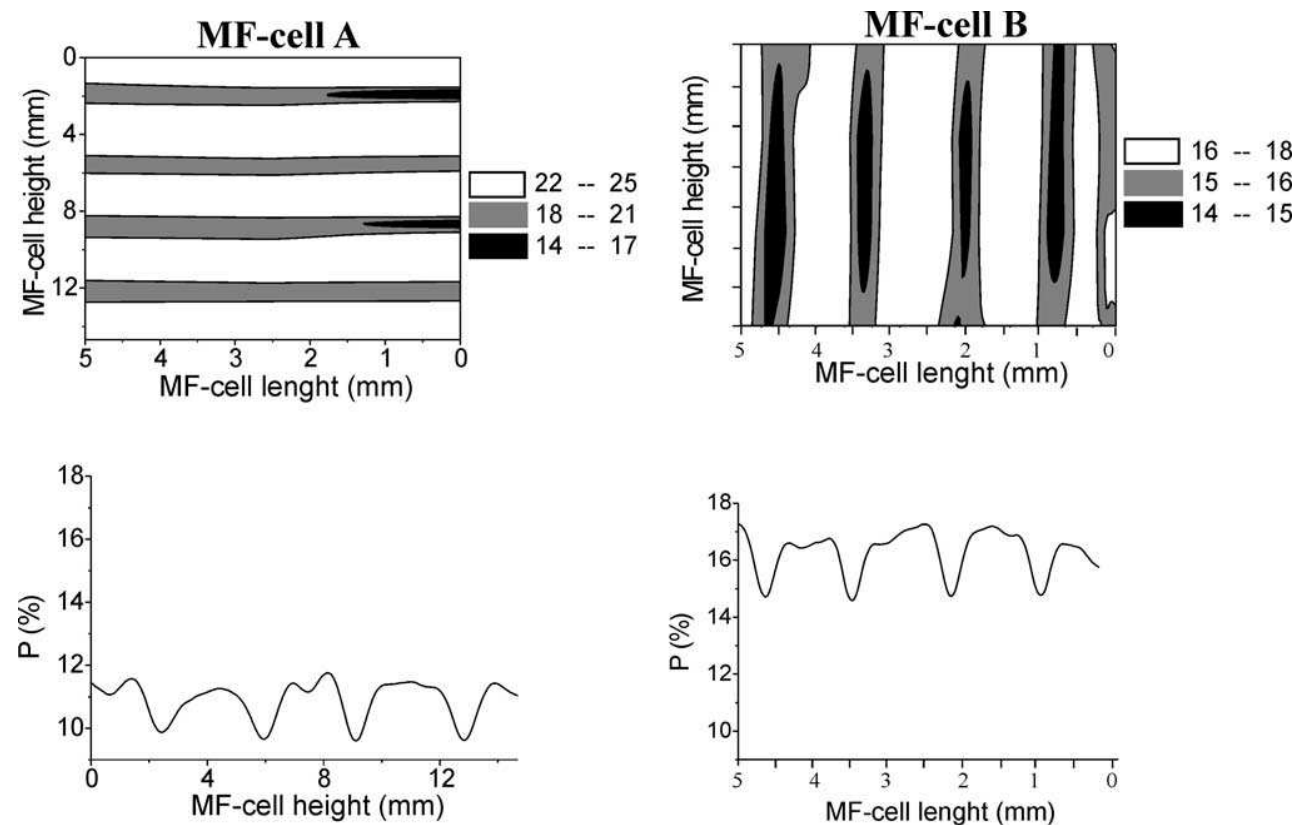

Fig. 3. Polarization maps (top) and typical profiles (bottom) within microchannel flow cells (MF cells). Right: $\mathrm{MF}$ cell $\mathrm{B}$, with $\mathrm{SnO} 2$ stripes on the inner wall parallel to the flow. Left: MF cell A, with $\mathrm{SnO}_{2}$ stripes on the inner wall perpendicular to the flow.

In both cases, polarization and anisotropy increased for the $\mathrm{SnO}_{2}$ regions on the inner walls. Essentially the same results, but with a much poorer signal-to-noise ratio, were obtained employing a CW Argon ion laser as the excitation source. This may be due to local heating of the sample under continuous incidence of the laser, resulting in a change in the ratio of probe rotational diffusion time and fluorescence lifetime.

There are three sources of polarization error in our system: the liquid input and output geometry, the pulsation of the peristaltic pump, and the laser intensity fluctuations. The first was due to the fact that the input and output are on the sides of the MF cells, generating a velocity gradient, and consequently, polarization increases from one side to the other. In the polarization maps, the effect of this gradient was removed from each polarization profile by a linear fit of the background and posterior subtraction as an offset. Although the laser pulses' intensity was not monitored, the fluctuation should be random and largely corrected for by the data averaging procedure. Peristaltic pump pulses added a constant $0.5-\mathrm{Hz}$ fluctuation to polarization which was removed by a $90-\mathrm{Hz}$ Fourier transform low-pass filter and posterior smoothing with a 5 point running average.

In order to determine PLF-FI reliability, peak widths for several different degrees of change in polarization were measured for flow parallel to the stripes of different chemical constitution $\left(W_{\text {II }}\right)$ and perpendicular to them $\left(W_{\perp}\right)$. As shown in Table $1, W_{\perp}$ had a greater error than $W_{\mathrm{II}}$, probably reflecting the greater variability associated with the flow across the molecular domains in the glass $/ \mathrm{SnO}_{2}$ transition region. A polarization decrease of $4 \%$ gave the best indication of the borosilicate stripe width for both flow orientations.
Table 1

Peak widths obtained for several degrees of polarization reduction $(\Delta P)$ for flow perpendicular $\left(W_{\perp}\right)$ and parallel $\left(W_{\mathrm{II}}\right)$ to the stripes on the inner wall of microchannel flow cells (MF cells)

\begin{tabular}{lrr}
\hline$\Delta P(\%)$ & \multicolumn{1}{c}{$W_{\perp}(\mu \mathrm{m})$} & \multicolumn{1}{c}{$W_{\text {II }}(\mu \mathrm{m})$} \\
\hline 0.1 & $(19 \pm 3) \times 100$ & $(17 \pm 1) \times 100$ \\
1.0 & $(16 \pm 2) \times 100$ & $(14 \pm 1) \times 100$ \\
2.0 & $(12 \pm 2) \times 100$ & $(11 \pm 1) \times 100$ \\
3.0 & $(8.5 \pm 0.5) \times 100$ & $(7.7 \pm 0.5) \times 100$ \\
4.0 & $(4 \pm 2) \times 100$ & $(5.2 \pm 0.5) \times 100$ \\
\hline
\end{tabular}

Assuming that the MF cells are slit channels [21], their Reynolds number will be below 2 and each boundary layer will persist for $69 \mu \mathrm{m}$, a value much larger than the channel width of $10 \mu \mathrm{m}$. Thus, the flow within the two MF cells consisted mainly of boundary layers. It is known that, in the boundary layer, there are greater interfacial interactions between fluid and walls. Since the inner walls of the cells had defined regions with two different chemical constitutions, the interfacial interactions were not the same over the face of the cells. The higher $\Gamma_{\mathrm{SL}}$ for MEG on glass points to greater interfacial adhesion, which in turn provokes a stronger drag on the flow, reducing the slip and decreasing the polarization. For $\mathrm{SnO}_{2}$, the lower $\Gamma_{\mathrm{SL}}$ implies weaker interfacial adhesion, with reduced drag at the interface and higher slip velocities of the liquid molecules.

From a molecular hydrodynamics approach, the transient intermolecular interactions between MEG and glass were of higher energy, while those between MEG and $\mathrm{SnO}_{2}$ were weaker, thus favoring molecular slip in the latter case. $\mathrm{SnO}_{2}$ aligned the molecular domains along the flow direction more effectively than glass. 


\section{Summary}

The absence of differences in polarization when the liquid flow is stopped as opposed to when it is flowing points to the different character of static and dynamic interfacial interactions.

Under flow, the relative molecular alignments of the fluorescent probe rhodamine $\mathrm{B}$, obtained by fluorescence depolarization, are in qualitative agreement with static interfacial tensions obtained from contact angle measurements. $\mathrm{SnO}_{2}$ regions had a lower liquid-solid interfacial interaction or adhesion and, consequently, produced higher polarization.

PLF-FI proved to be independent of flow orientation parallel and perpendicular to the $\mathrm{SnO}_{2}$ stripes, although the precision was greater for the former.

Although the detection is external, it is a reliable method for thin cells where the flow is strongly affected by the interfacial interactions that vary with the chemical constitution of the inner walls.

PLF-FI has been shown to be a useful nondestructive method for evaluating local changes of chemical constitution of the inner walls of MF cells.

\section{Acknowledgments}

The authors thank the Conselho Nacional de Desenvolvimento Científico e Tecnológico (CNPq, Brazil) for grants and the Ibero-American Network of Liquid Crystal Displays, SubProgram IX: Microelectronics of Program CYTED for their support for this work. A.M.V.L. acknowledges an undergraduate research fellowship support from PIBIC-CNPq and CNPq. Y.N.M. acknowledges a CNPq doctoral scholarship. C.M.Q. acknowledges a senior research scholarship from CNPq.

\section{References}

[1] G.M. Whitesides, A.D. Stroock, Phys. Today 54 (2001) 42.

[2] A.W. Adamson, A.P. Gast, Physical Chemistry of Surfaces, 6th ed., Wiley, New York, 1997.

[3] C.W. Extrand, J. Colloid Interface Sci. 248 (2002) 136, doi:10.1006/ jcis.2001.8172.

[4] C.M. Quintella, Â.M.V. Lima, C.C. Gonçalves, Y.N. Watanabe, M.A. Schreiner, A.P. Mammana, I. Pepe, A.M. Pizzo, J. Colloid Interface Sci. 262 (2003) 221.

[5] C.M. Quintella, C.C. Gonçalves, I. Pepe, A.M.V. Lima, A.P.S. Musse, J. Braz. Chem. Soc. 12 (2001) 780, available on line, in English, at http://jbcs.sbq.org.br/jbcs/2001/vol12_n6/14.pdf.

[6] P.P. Feofilov, The Physical Basis of Polarized Emission, Consultants Bureau, New York, 1961.

[7] A. Kawski, Crit. Rev. Anal. Chem. 23 (1993) 459.

[8] J.R. Lakowicz, Principles of Fluorescence Spectroscopy, Plenum, New York/London, 1983.

[9] G.J. Juzeliunas, J. Lumin. 46 (1990) 201.

[10] A.J. Kenyon, A.J. McCaffery, C.M. Quintella, Mol. Phys. 72 (1991) 965.

[11] A.J. Kenyon, A.J. McCaffery, C.M. Quintella, J.F. Winkel, Mol. Phys. 74 (1991) 871.

[12] A.J. Bain, P. Chandna, G. Butcher, Chem. Phys. Lett. 260 (1996) 441.

[13] C.M. Quintella, A.P.S. Musse, C.C. Gonçalves, A.J. McCaffery, Exp. Fluids 35 (2003) 41.

[14] V. Baranauskas, Z. Jingguo, A.P. Mammana, T.E.A. Santos, M.A. Schreiner, C.I.Z. Mammana, Sens. Actuat. A 85 (2002) 90.

[15] P. Bojarski, A. Jankowicz, J. Lumin. 81 (1999) 21.

[16] M. Megens, R. Sprik, G.H. Wegdam, A. Lagendijk, J. Chem. Phys. 107 (1997) 493.

[17] A.D. Kummer, C. Kompa, H. Niwa, T. Hirano, S. Kojima, M.E. Michel-Beyerle, J. Phys. Chem. B 106 (2002) 7554.

[18] G.B. Dutt, S. Doraiswamy, N. Periasamy, B. Venkataraman, J. Chem. Phys. 93 (1990) 8498.

[19] A.D. Scully, A. Matsumoto, S. Hirayama, Chem. Phys. 157 (1991) 253.

[20] C.M. Quintella, C.C. Gonçalves, I. Pepe, A.M.V. Lima, A.P.S. Musse, J. Automat. Methods Manage. Chem. 24 (2002) 31, available online in English at http://taylorandfrancis.metapress.com.

[21] H. Härri, S. Leutwyler, E. Schumacher, Rev. Sci. Instrum. 53 (1982) 1855. 